\title{
A Versatile Polymer Micelle Drug Delivery System for Encapsulation and In Vivo Stabilization of Hydrophobic Anticancer Drugs
}

\author{
Jonathan Rios-Doria, ${ }^{1}$ Adam Carie, ${ }^{1}$ Tara Costich, ${ }^{1}$ Brian Burke, ${ }^{1}$ Habib Skaff, ${ }^{1}$ \\ Riccardo Panicucci, ${ }^{2}$ and Kevin Sill ${ }^{1}$ \\ ${ }^{1}$ Intezyne Inc., 3720 Spectrum Boulevard, Suite 104, Tampa, FL 33612, USA \\ ${ }^{2}$ Novartis Institutes for BioMedical Research, Cambridge, MA 02139, USA \\ Correspondence should be addressed to Kevin Sill, kevin.sill@intezyne.com
}

Received 27 July 2011; Accepted 15 October 2011

Academic Editor: Eliana B. Souto

Copyright ( $\odot 2012$ Jonathan Rios-Doria et al. This is an open access article distributed under the Creative Commons Attribution License, which permits unrestricted use, distribution, and reproduction in any medium, provided the original work is properly cited.

\begin{abstract}
Chemotherapeutic drugs are widely used for the treatment of cancer; however, use of these drugs is often associated with patient toxicity and poor tumor delivery. Micellar drug carriers offer a promising approach for formulating and achieving improved delivery of hydrophobic chemotherapeutic drugs; however, conventional micelles do not have long-term stability in complex biological environments such as plasma. To address this problem, a novel triblock copolymer has been developed to encapsulate several different hydrophobic drugs into stable polymer micelles. These micelles have been engineered to be stable at low concentrations even in complex biological fluids, and to release cargo in response to low $\mathrm{pH}$ environments, such as in the tumor microenvironment or in tumor cell endosomes. The particle sizes of drugs encapsulated ranged between $30-80 \mathrm{~nm}$, with no relationship to the hydrophobicity of the drug. Stabilization of the micelles below the critical micelle concentration was demonstrated using a pH-reversible crosslinking mechanism, with proof-of-concept demonstrated in both in vitro and in vivo models. Described herein is polymer micelle drug delivery system that enables encapsulation and stabilization of a wide variety of chemotherapeutic drugs in a single platform.
\end{abstract}

\section{Introduction}

It was estimated that there were $1,500,000$ new cancer cases and approximately 560,000 deaths from cancer in 2010 [1]. The use of chemotherapy has dramatically improved the survival rate of patients for the last several decades; however, stand-alone chemotherapy drugs suffer from numerous problems including rapid in vivo metabolism and/or excretion, inability to access and penetrate cancer cells, and nonspecific uptake by healthy cells and tissue. Often, a large percentage of cytotoxic drug administered to the patient does not reach the tumor environment but rather is distributed throughout the body, resulting in the many toxic effects associated with chemotherapy and a narrowing of the drug's therapeutic window. Polymer micelles offer a promising approach to achieving these goals due to their inherent ability to overcome multiple biological barriers, such as avoidance of the reticuloendothelial system (RES) [2]. Due to their unique size range $(20-150 \mathrm{~nm})$, micelles are able to avoid renal clearance (typically less than $20 \mathrm{~nm}$ ) and uptake by the liver and spleen (particles greater than $150 \mathrm{~nm}$ ). These micelles can also preferentially accumulate in solid tumors via the enhanced permeation and retention (EPR) effect $[3,4]$. The EPR effect is a consequence of the disorganized nature of the tumor vasculature, which results in increased permeability of polymer therapeutics and drug retention at the tumor site.

When considering the design of a nanocarrier, several important factors should be addressed. An ideal delivery system should be composed of biocompatible and biodegradable materials, reproducibly assemble into the desired size range, encapsulate a wide range of drugs and drug classes, 
maintain particle size in biological media, have the ability to attach cell-specific targeting groups, and release the therapeutic at the site of disease. Polymer micelles have received much attention over the past thirty years as drug delivery vehicle [5-11]. In traditional micelle systems, however, there are no mechanisms in place to keep the micelle intact when it is diluted in the bloodstream, where it is below the critical micelle concentration and interacts with surfactant proteins within the blood. Thus, stability of nano-carriers in biological media remains an issue that needs to be addressed [12]. Some have utilized the approach of chemically conjugating the active drug to a polymer to potentially improve stability. However, this "prodrug" approach is dependent on enzymatic or chemical cleavage of the bond to release the active drug [13-15]. In an attempt to add stability to the micelle, various types of micelles have been developed whereby either the core or shell of the micelle has incorporated crosslinking chemistries, thereby imparting stability at low micelle concentrations [16-22]. However, in many cases, crosslinking is achieved utilizing covalent bonding within the micelle, which does not lend itself to tunable drug release. In addition, in some crosslinked micelles, the crosslinks are physically located with the drug in the core of the micelle, which may interfere with pharmaceutical drug action or drug release from the micelle.

This paper describes a polymer micelle drug delivery system (IVECT) that has effectively addressed the limitations of traditional polymer micelles, by forming micelles that are stable in biological environments. The IVECT triblock copolymer consists of poly(ethylene glycol)- $b$-poly(aspartic acid)- $b$-poly(D-leucine-co-tyrosine). The leucine/tyrosine core unit in this polymer is able to encapsulate a wide variety of hydrophobic molecules, which is enhanced by the use of both D and L stereoisomers. The poly(aspartic acid) block was designed to participate in a metal-acetate crosslinking reaction that effectively stabilized drugs inside the core of the micelle and also mediates $\mathrm{pH}$-dependent release of the drug. In this paper, a polymer micelle is described that is composed of biocompatible materials, has the versatility to encapsulate a wide range of therapeutic payloads, is stable to dilution within the blood stream, and has a tunable, highly sensitive, and reversible stabilization mechanism. Data are presented whereby several different hydrophobic molecules are encapsulated and stabilized by crosslinking using a single polymer and without physical manipulation of the drug.

\section{Materials and Methods}

2.1. Chemicals and Reagents. All chemicals were obtained from Aldrich or Fisher unless otherwise specified. $\mathrm{N}_{3}$ PEG12k-NH-BOC was prepared as described previously [23]. N-carboxy anhydrides (NCAs) were prepared according to previously published procedures. [24, 25]. N-methylpyrrolidone (NMP) was distilled prior to use. BB4007431 and NX-8 were provided by Novartis. Daunorubicin and doxorubicin were obtained from LGM Pharma (Boca Raton, FL). All other drugs were obtained from Yingxuan Pharmaceuticals (Shanghai, China).
2.2. Synthesis of Triblock Copolymer. $\mathrm{N}_{3}$-PEG12K-NH-Boc (150 g, $12.5 \mathrm{mmol}$ ) was dissolved into $1 \mathrm{~L}$ of $\mathrm{CH}_{2} \mathrm{Cl}_{2} / \mathrm{DFA}$ (70/30) and was allowed to stir at room temperature overnight. The product was precipitated twice in diethyl ether and was recovered as a white powder (yield 90\%). ${ }^{1} \mathrm{H}$ NMR (d $\mathrm{d}_{6}$-DMSO) $7.77(3 \mathrm{H}), 5.97(1 \mathrm{H}), 3.83-3.21(1050 \mathrm{H}), 2.98$ (2 H) ppm.

$\mathrm{N}_{3}$-PEG12K-NH $3 /$ DFA (95 g, $7.92 \mathrm{mmol}$ ) was weighed into an oven-dried, 2 L-round-bottom flask and was left under vacuum for three hours before adding the NCA. Asp $(\mathrm{OBu}) \mathrm{NCA}(17.04 \mathrm{~g}, 79.2 \mathrm{mmol}$ ) was added to the flask, and the flask was evacuated under reduced pressure and subsequently backfilled with nitrogen gas. Dry NMP $(560 \mathrm{~mL})$ was introduced by cannula, and the solution was heated to $60^{\circ} \mathrm{C}$. The reaction mixture was allowed to stir for 24 hours at $60^{\circ} \mathrm{C}$ under nitrogen gas. Then, D-Leu NCA (24.88 g, $158 \mathrm{mmol}$ ) and Tyr (OBzl) NCA (47.08 g, $158 \mathrm{mmol})$ were dissolved under nitrogen gas into $360 \mathrm{~mL}$ of NMP into an oven-dried, round-bottom flask, and the mixture was subsequently added to the polymerization reaction via a syringe. The solution was allowed to stir at $60^{\circ} \mathrm{C}$ for another three days at which point the reaction was complete (as determined by HPLC). The solution was cooled to room temperature, and diisopropylethylamine (DIPEA) (10 mL), dimethylaminopyridine (DMAP) (100 mg), and acetic anhydride $(10 \mathrm{~mL})$ were added. Stirring was continued for 1 hour at room temperature. The polymer was precipitated into diethyl ether $(10 \mathrm{~L})$ and isolated by filtration. The solid was redissolved in dichloromethane $(500 \mathrm{~mL})$ and precipitated into diethyl ether $(10 \mathrm{~L})$. The product was isolated by filtration and dried in vacuo to give the block copolymer as an offwhite powder $(134.6 \mathrm{~g}$, yield $=73 \%) .{ }^{1} \mathrm{H}$ NMR $\left(\mathrm{d}_{6}\right.$-DMSO $)$ $\delta$ 8.43-7.62 $(50 \mathrm{H}), 7.35(100 \mathrm{H}), 7.1(40 \mathrm{H}), 6.82(40 \mathrm{H})$, $4.96(40 \mathrm{H}), 4.63-3.99(50 \mathrm{H}), 3.74-3.2(1500 \mathrm{H}), 3.06-2.6$ $(60 \mathrm{H}), 1.36(90 \mathrm{H}), 1.27-0.47(180)$.

$\mathrm{N}_{3}$-PEG12K- $b$-poly $\left(\mathrm{Asp}(\mathrm{OBu})_{10}\right)-b$-poly $\left(\operatorname{Tyr}(\mathrm{OBzl})_{20^{-}}\right.$ co-D-Leu 20 )-Ac (134.6 g, $6.4 \mathrm{mmol})$ was dissolved into $1 \mathrm{~L}$ of a solution of pentamethylbenzene (PMB, $0.5 \mathrm{M}$ ) in trifluoroacetic acid (TFA). The reaction was allowed to stir for five hours at room temperature. The solution was precipitated into a 10-fold excess of diethyl ether, and the solid was recovered by filtration. The polymer was redissolved into $800 \mathrm{~mL}$ of dichloromethane and precipitated into diethyl ether. An off-white polymer was obtained after drying the product overnight in vacuo $(111.8 \mathrm{~g}$, yield $=93 \%)$. ${ }^{1} \mathrm{H} \mathrm{NMR}\left(\mathrm{d}_{6}\right.$ DMSO) $\delta 12.2(10 \mathrm{H}), 9.1(10 \mathrm{H}), 8.51-7.71(50 \mathrm{H}), 6.96$ $(40 \mathrm{H}), 6.59(40 \mathrm{H}), 4.69-3.96(60 \mathrm{H}), 3.81-3.25(1500 \mathrm{H})$, 3.06-2.65 (60 H), 1.0-0.43 (180). ${ }^{1} \mathrm{H}$ NMR ( $\left.\mathrm{d}_{6}-\mathrm{DMSO}\right) \delta$ $171.9,171,170.5,170.3,155.9,130.6,129.6,127.9,115.3$, $114.3,70.7,69.8,54.5,51.5,50,49.8,49.4,36.9,36,24.3$, 23.3, 22.3, 21.2. IR (ATR) 3290, 2882, 1733, 1658, 1342, $1102,962 \mathrm{~cm}^{-1}$. The final composition of the polymer is $\mathrm{N}_{3}$-PEG12K- $b$-poly(Asp) ${ }_{10}-b$-poly $\left(\mathrm{Tyr}_{20}-c o-\mathrm{D}-\mathrm{Leu}_{20}\right)-\mathrm{Ac}$, which is also referred to as poly(ethylene glycol)- $b$-poly (aspartic acid)- $b$-poly(D-leucine-co-tyrosine).

2.3. Micelle Production. All formulations were prepared using oil-in-water emulsion techniques involving dissolving 
the polymer in water and the drug in an organic solvent. An exemplary formulation technique for daunorubicin follows. The IVECT triblock copolymer $(3 \mathrm{~g})$ was dissolved in wa-ter $(500 \mathrm{~mL})$. Daunorubicin $(301 \mathrm{mg})$ was dissolved in dichloromethane $(48 \mathrm{~mL})$ and methanol $(12 \mathrm{~mL})$. Just prior to use, triethylamine $(0.28 \mathrm{~mL})$ was added to the organic solution to complete the dissolution of the daunorubicin. The aqueous solution was mixed with a Silverson LRT- 4 shear mixer (fine emulsor screen, 10,000 RPM). Daunorubicin was added to the mixed solution in a single portion over $\sim 10 \mathrm{~s}$. The solution was mixed for an additional minute and then stirred at room temperature overnight. The resulting solution was then filtered through a $0.22 \mu \mathrm{m}$ PES filter (Millipore Stericup). Iron (II) chloride solution was added to the concentrated micelle solution at a concentration of $10 \mathrm{mM}$, and the $\mathrm{pH}$ was adjusted to 8.0 and stirred overnight. This solution was frozen on a shell freezer at $-40^{\circ} \mathrm{C}$ and then lyophilized on a Labconco $6 \mathrm{~L}$ Plus manifold lyophilization system operating at a pressure of 0.050 Torr and a collector temperature of $-85^{\circ} \mathrm{C}$. After $48 \mathrm{~h}$, crosslinked, daunorubicin-loaded micelles were recovered as a purple powder $(3.22 \mathrm{~g}, 93 \%$ yield).

2.4. Drug Weight Loading by HPLC. The mass percentage of active drug within the formulation was determined by HPLC. An exemplary procedure for daunorubicin follows. The daunorubicin-loaded micelle was analyzed by a Waters Alliance separations module (W2695) equipped with Waters Novapak C18, $4 \mu \mathrm{m}$ column (no. WAT086344) coupled with a Waters Photodiode Array Detector (W2998). Daunorubicin was detected at an absorbance of $480 \mathrm{~nm}$. Mobile phase consisted of a $10: 70: 20$ ratio of methanol: $10 \mathrm{mM}$ phosphate buffer $\mathrm{pH} 2.0$ :acetonitrile over a 10-minute gradient. Known standards of free daunorubicin were used to determine the percentage by weight of daunorubicin in the formulation (wt/wt\%).

2.5. Particle Size Analysis. Particle sizes were determined using dynamic light scattering on a Wyatt DynaPro (Santa Barbara, CA). Following lyophilization, micelles were dissolved at $1 \mathrm{mg} / \mathrm{mL}$ in $150 \mathrm{mM} \mathrm{NaCl}$ and were centrifuged at 2,000 RPM prior to analysis to remove dust.

2.6. Encapsulation, Crosslinking, and pH-Dependent Release Dialysis. To test drug encapsulation, the uncrosslinked formulation was dissolved at a concentration of $20 \mathrm{mg} / \mathrm{mL}$ in water, which is above the critical micelle concentration of the polymer. Two milliliters were dialyzed in a $3500 \mathrm{MWCO}$ dialysis bag in a volume of $300 \mathrm{~mL}$ of $10 \mathrm{mM}$ phosphate buffer, $\mathrm{pH}$ 8.0. After dialysis for six hours, the pre- and postdialysis samples from inside the bag were quantified for drug concentration by HPLC. Encapsulation retention was calculated by dividing the postdrug concentration by the preconcentration.

To test crosslinking, the crosslinked formulation was dissolved in water at a concentration of $0.2 \mathrm{mg} / \mathrm{mL}$, which is below the critical micelle concentration. Three milliliters were dialyzed in a $3500 \mathrm{MWCO}$ dialysis bag in a volume of $300 \mathrm{~mL}$ of $10 \mathrm{mM}$ phosphate buffer $\mathrm{pH}$ 8. After dialysis for six hours, the pre- and postdialysis samples from inside the bag were quantified for drug concentration by HPLC. Crosslinking retention was calculated by dividing the postdrug concentration by the preconcentration. For $\mathrm{pH}$-dependent release, samples were treated the same as for crosslinking dialysis except for dialysis in $10 \mathrm{mM}$ phosphate buffer pH 3, 4, 5, 6, 7, 7.4, or 8 .

2.7. In Vivo Pharmacokinetic Studies. Female Sprague-Dawley rats weighing about $220 \mathrm{~g}$ with jugular vein catheters were obtained from Harlan. Rats were randomly divided into groups of four and were given a single injection of free drug, uncrosslinked drug loaded micelles, or crosslinked, drug loaded micelles dissolved in $150 \mathrm{mM} \mathrm{NaCl}$. Daunorubicin micelles were injected at $10 \mathrm{mg} / \mathrm{kg}$ daunorubicin-equivalent dosing, and BB4007431 micelles were injected through the catheter at $25 \mathrm{mg} / \mathrm{kg}$ BB4007431 drug-equivalent dosing. Free BB4007431 was dissolved in $0.33 \mathrm{M}$ lactic acid/1.67\% dextrose and then diluted in $5 \%$ dextrose in water for injection. About $0.25 \mathrm{~mL}$ of blood was collected through the catheter at 1, 5, $15 \mathrm{~min}, 1 \mathrm{~h}, 4 \mathrm{~h}, 8 \mathrm{~h}$, and $24 \mathrm{~h}$. Samples were centrifuged at 2000 RPM for 5 minutes to separate plasma. Plasma was then diluted $1: 4$ in cold $0.1 \%$ phosphoric acid in methanol with an appropriate internal standard, vortexed for 10 minutes, and centrifuged for 13,000 RPM for 10 minutes. The supernatant was then analyzed by HPLC to determine the drug concentration for each sample. Plasma concentrations were plotted in Microsoft Excel to determine AUC values. Animals were maintained in accordance with The Public Health Service Policy on Humane Care and Use of Laboratory Animals, and the Institutional Animal Care and Use Committee's (IACUC) Principles and Procedures of Animal Care and Use.

\section{Results}

The IVECT triblock copolymer consists of poly(ethylene glycol)- $b$-poly(aspartic acid)- $b$-poly(D-leucine-co-tyrosine), in which each segment is biodegradable or biocompatible and plays a very important role (Figure 1). Hydrophobic drugs that are loaded into the micelle reside in the encapsulation block (yellow), forming the core of the micelle. The poly(aspartic acid) middle block (green) is the crosslinking block that stabilizes the micelle. In contrast to crosslinking in the core or periphery of the micelle, Intezyne has developed $\mathrm{pH}$-reversible crosslinking technology in the middle block of the triblock copolymer. Crosslinking of this middle layer of the micelle is advantageous since it does not interfere with the core region, which is where the drug resides. The chemistry utilized to crosslink the polymer chains together, and thus stabilizes the micelle, is based on metal acetate chemistry (Figure 2). It is well known that a number of metal ions can interact with carboxylic acids to form metal-acetate bonds [26]. It is also understood that these ligation events form rapidly when the carboxylic acid is in the carboxylate form (e.g., high $\mathrm{pH}, \mathrm{pH} \sim 7-8$ ) yet only weakly interact when the carboxylic acids are fully protonated (e.g., low 


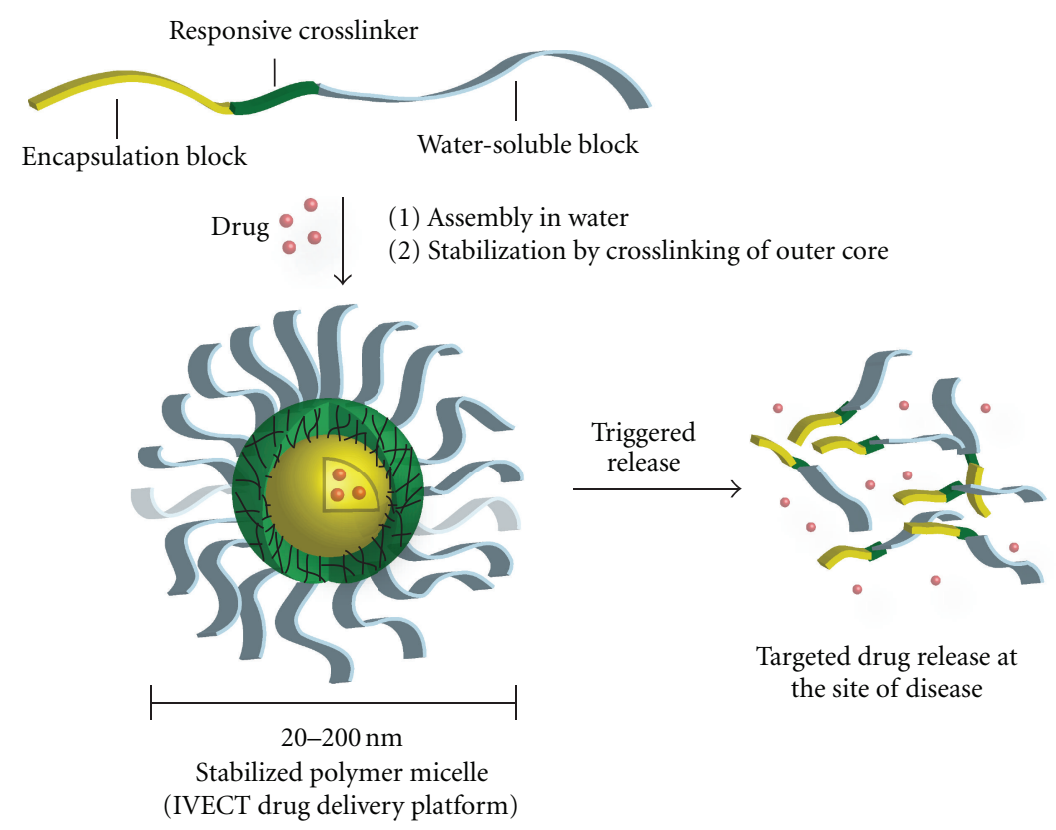

FIGURE 1: The IVECT polymer micelle. Drugs are loaded into the core hydrophobic block (yellow). The crosslinking block (green) provides stability to the micelle by forming $\mathrm{pH}$-reversible metal-acetate bonds that allow for triggered drug release near the tumor. The PEG block (gray) gives the micelle aqueous solubility and stealth properties in vivo.

$\mathrm{pH}, \mathrm{pH} 4-5$ ), therefore allowing release of the drug in low$\mathrm{pH}$ environments, such as regions surrounding the tumor, and the endosomes of tumor cells following endocytosis of micelles. The poly(ethylene glycol) block (Figure 1, shown in gray) allows for water solubility and provides "stealth" properties to the micelle in order to avoid protein opsonization and the reticuloendothelial system [2].

As an initial study, the triblock copolymer was used to encapsulate several different small molecule drugs with varying hydrophobicities. A trend was discovered such that the ability of the triblock to encapsulate a drug was dependent on the drug's $\log P$ value. Effective encapsulation was achieved with molecules having a $\log P>1.4$ (Figure 3). The weight loadings of the formulations ranged between 1 and $20 \%$. Molecules that were encapsulated were subsequently crosslinked by the addition of iron chloride. The addition of iron chloride to the micelle did not affect the drug and did not result in generation of polymer-drug conjugates. To test stability of the crosslinked micelle, the in vitro stability of the micelle below the CMC was determined using a dialysis assay. In contrast to the encapsulation retention, there was no clear correlation between the $\log P$ value and crosslinking retention (Table 1). The particle sizes of crosslinked micelles, as determined by dynamic light scattering, also did not seem related to the $\log P$ value. These results demonstrate that the hydrophobicity of the drug influences its ability to be encapsulated within the micelle, but does not influence crosslinking retention or particle size.

To determine whether crosslinked micelles exhibited $\mathrm{pH}$ dependent release, different micelles were dialyzed at concentrations below the CMC in $10 \mathrm{mM}$ phosphate buffer of different pHs. Crosslinked micelles containing BB4007431
TABLE 1: Drug formulation properties. The encapsulation retention percentage, crosslinking retention percentage, and particle sizes are shown for eleven compounds tested for loading within the polymer micelle.

\begin{tabular}{lcccc}
\hline Drug & Log $P$ & $\begin{array}{c}\text { Encapsulation } \\
\text { retention (\%) }\end{array}$ & $\begin{array}{c}\text { Crosslinking } \\
\text { retention (\%) }\end{array}$ & $\begin{array}{c}\text { Particle size } \\
(\mathrm{nm})\end{array}$ \\
\hline 5-Fluorouracil -0.58 & 0 & $\mathrm{NA}$ & $\mathrm{ND}$ \\
Caffeine & -0.24 & 0 & $\mathrm{NA}$ & $\mathrm{ND}$ \\
Melphalan & -0.22 & 0 & $\mathrm{NA}$ & $\mathrm{ND}$ \\
Gemcitabine & 0.14 & 0 & $\mathrm{NA}$ & $\mathrm{ND}$ \\
Etoposide & 0.73 & 12 & $\mathrm{NA}$ & $\mathrm{ND}$ \\
Doxorubicin & 1.41 & 80 & 63 & 30 \\
Daunorubicin & 1.68 & 85 & 78 & 30 \\
BB4007431 & 1.94 & 79 & 90 & 55 \\
Paclitaxel & 3.2 & 93 & 60 & 36 \\
NX-8 & 4.18 & 86 & 52 & 86 \\
Vinorelbine & 4.39 & 87 & 37 & 47 \\
\hline
\end{tabular}

NA: not applicable, ND: not determined.

demonstrated $\mathrm{pH}$-dependent release of the drug, with increased retention of the drug within the micelle at $\mathrm{pH} 8$, and near total release of the drug after incubation at $\mathrm{pH} 3$ (Figure 4(a)). In contrast, uncrosslinked micelles containing BB4007431 showed nearly complete release of the drug at all $\mathrm{pHs}$, reflecting the instability of the uncrosslinked micelle. To assess the effect of salt in the stability of the micelle, crosslinked BB4007431 was diluted below the CMC and dialyzed in $10 \mathrm{mM}$ phosphate buffer or phosphate-buffered saline (PBS) at different pHs (Figure 4(b)). This experiment 


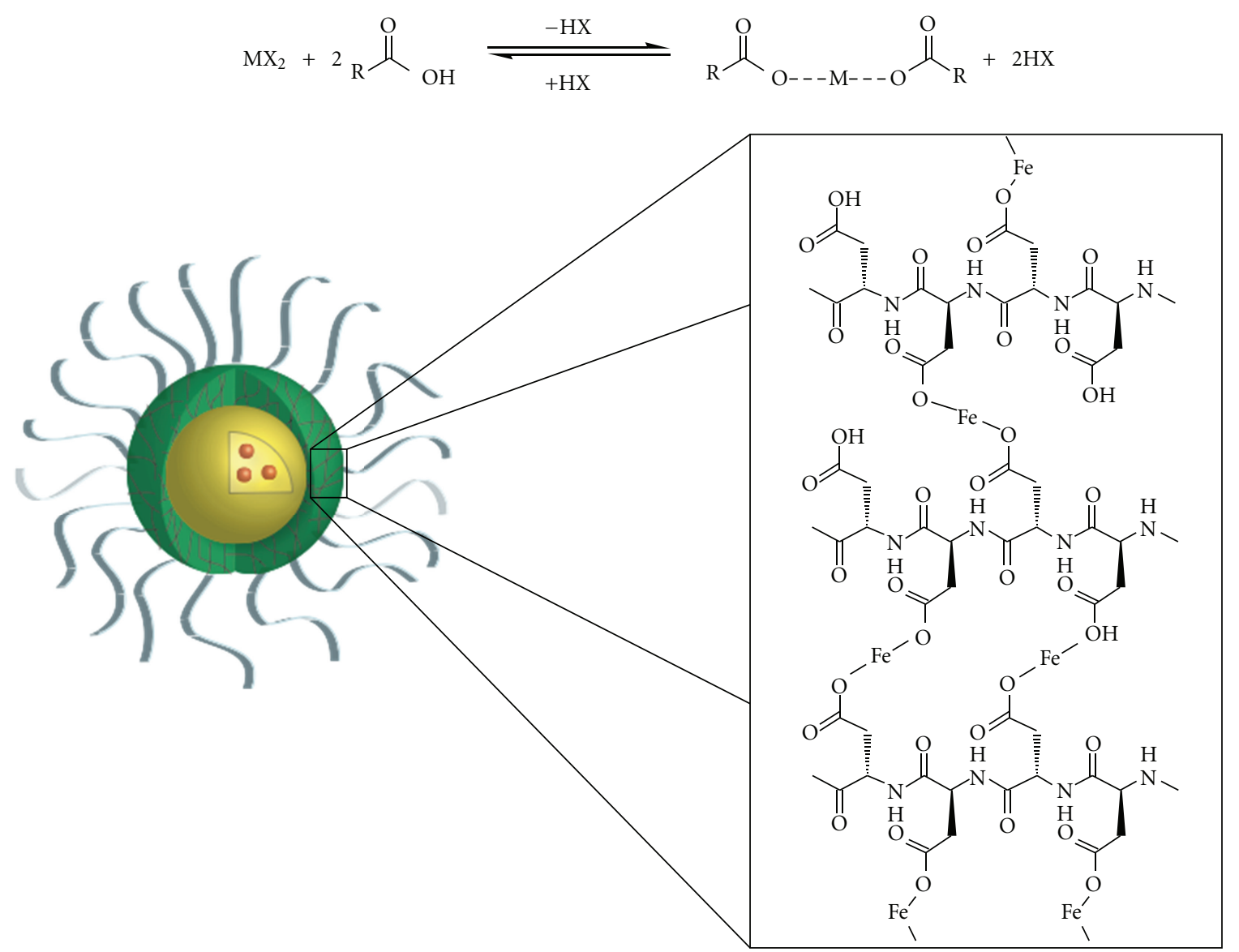

Figure 2: Metal-acetate crosslinking chemistry for stabilization of polymer micelles. While the drug is localized in the core block, the poly(aspartic acid) block of the middle block reacts with metals to form metal acetate bonds. Bonds are formed at high $\mathrm{pH}$ and are dissociated at low $\mathrm{pH}$. M represents metal, and X represents a halogen.

showed that salt did destabilize the crosslinked micelle to some degree, but a pH-dependent release was still exhibited.

In order to test the stability of the micelle in vivo, a crosslinked, daunorubicin-loaded micelle was assessed in a pharmacokinetic study. Rats were intravenously injected with $10 \mathrm{mg} / \mathrm{kg}$ of free daunorubicin, uncrosslinked daunorubicin micelle, or crosslinked daunorubicin micelle, and the concentration of daunorubicin in plasma was determined over the course of twenty four hours (Figure 5). Results demonstrated that the crosslinked daunorubicin micelle exhibited 90-fold increase in plasma in AUC compared to free daunorubicin and 78-fold increase in AUC compared to uncrosslinked daunorubicin. Crosslinked daunorubicin also exhibited a 46 -fold higher $C_{\max }$ than free daunorubicin and a 59-fold increase compared to uncrosslinked micelle. These data demonstrate significantly higher in vivo micelle stability with the crosslinked daunorubicin micelle compared to the free drug. A similar study was repeated with a crosslinked formulation of compound BB4007431. Rats injected with crosslinked BB4007431 micelle displayed a vastly superior increase in $C_{\max }$ (20-fold) and AUC (202.4-fold) compared to free drug (Figure 6). Similar increases in stability were also obtained with crosslinked doxorubicin and paclitaxel-loaded micelles (data not shown), demonstrating the wide applicability of this crosslinking technology to provide increased drug stability in vivo.

\section{Discussion}

Improving stability of therapeutic molecules is a well-established aim in the field of drug delivery. An ideal drug-loaded nanoparticle would be stable to dilution in biological media, possess stealth-like properties to avoid uptake by the RES, and release the drug only in the area of diseased tissue. The data presented in this paper describe a versatile polymer micelle drug delivery system that has been engineered to efficiently encapsulate a wide variety of hydrophobic drugs. In addition, the stabilization technology built-in to the micelle is dependent on $\mathrm{pH}$, such that the micelle is stable at physiological $\mathrm{pH}$, and unstable at low $\mathrm{pH}$, thus providing a mechanism to release the drug in the tumor microenvironment or in endosomes, which are both slightly acidic environments.

A vast number of drugs exist today that possess potent anticancer activity; however, many of them are unable to be utilized in the clinic due to their inability to be dissolved 


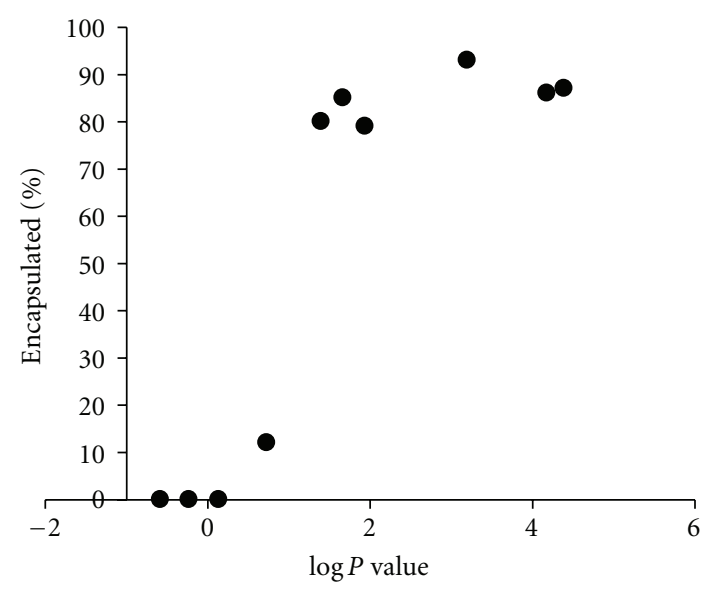

FIGURE 3: Encapsulation retention of drugs within the micelle is correlated to $\log P$ value. The encapsulation retention of the drug, based on an in vitro dialysis assay, is plotted compared to its $\log P$ value.

in aqueous solutions [27]. Some hydrophobic drugs can be solubilized with excipients; however, such vehicles have been shown to cause toxicity to the patient [28]. The core block of the triblock copolymer (poly(D-leucine-co-tyrosine)) was rationally designed and chosen to encapsulate hydrophobic molecules. A key factor leading to the versatility arises from the use of both D and L stereoisomers of amino acids in the core block, which disrupts the secondary structure of the polypeptide. Replacing the rod-like helical nature of the polypeptide with the flexibility of a random coil allows for significant increases in drug loading efficiency. The ability of drugs to be encapsulated within the triblock copolymer was related to its $\log P$ value, such that only hydrophobic drugs could be encapsulated. This result is logical as hydrophilic molecules would prefer to associate with the hydrophilic part of the polymer versus the hydrophobic core, leading to inefficient drug encapsulation.

Crosslinking was performed using metal acetate chemistry, specifically, iron (II) chloride. The crosslinking dialysis assay determined that $40-90 \%$ of the drug remained in the crosslinked micelle after six hours. Typically, $10 \%$ of the drug or less was retained in uncrosslinked micelles examined using the same crosslinking dialysis assay. Although there was a correlation between $\log P$ and encapsulation ability, there was no clear correlation between $\log P$ and the crosslinking retention or the particle size. Therefore, it is hypothesized that while hydrophobicity is a strong predictor of success for encapsulation, other variables such as chemical functionality and drug crystallinity play a significant role in micelle size and crosslinking efficiency.

While stability is important, equally important is the ability to release the drug in a controlled fashion at the site of disease. In vitro release assays demonstrated progressive release of drug from the core of the micelle as the $\mathrm{pH}$ decreased, which has physiological relevance for delivering drugs to tumors. While passive targeting of nanoparticles within tumor tissue is accomplished by the EPR effect, an additional

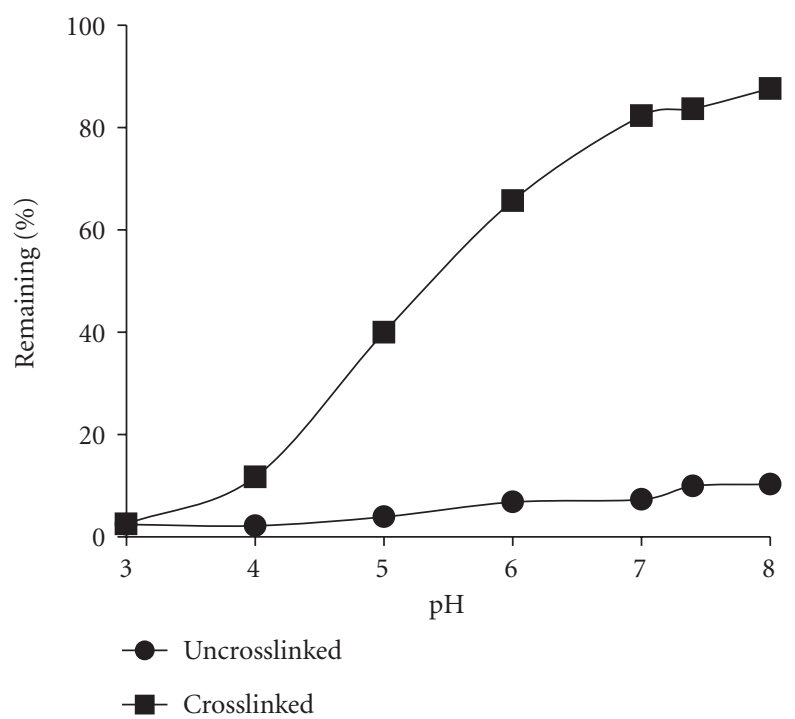

(a)

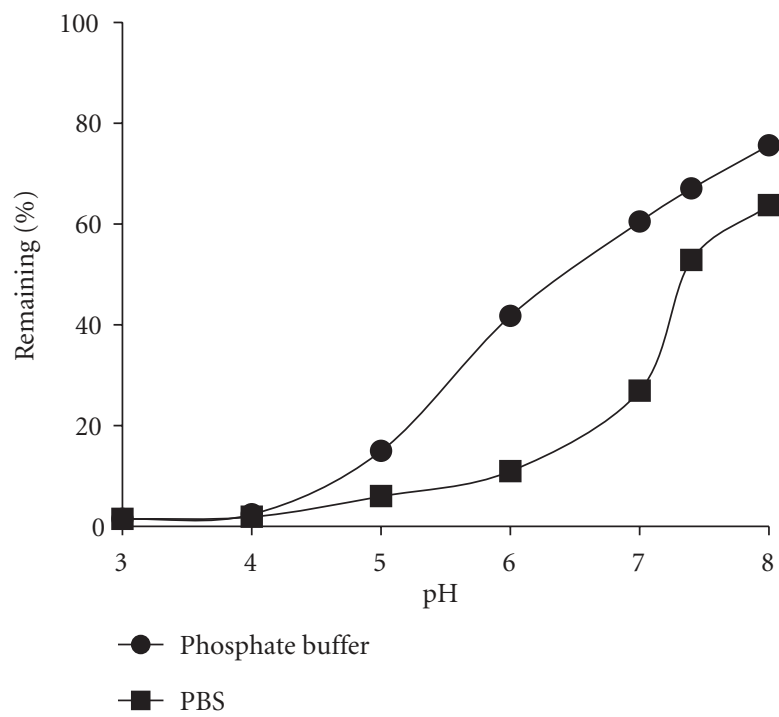

(b)

FIGURE 4: pH-dependent release of drug-loaded micelles. (a) Crosslinked and uncrosslinked BB4007431 micelles were diluted below the $\mathrm{CMC}$ and dialyzed for 6 hours in $10 \mathrm{mM}$ phosphate buffer at different pHs. The amount of drug retained before and after dialysis was quantified by HPLC. (b) Crosslinked BB4007431 micelles were diluted below the CMC and dialyzed for 6 hours in either $10 \mathrm{mM}$ phosphate buffer, or PBS, at different pHs. Drug content remaining was quantified by HPLC as above.

layer of targeting is possible by employing active targeting strategies, such as decorating the surface of nanoparticles with targeting ligands [29-33]. It is logical to conclude, however, that the ability to target a nanoparticle to tumors is dependent on the stability of the nanoparticle in vivo. In pharmacokinetic experiments, superior AUC and $C_{\max }$ were obtained with several crosslinked micelles, including daunorubicin and BB4007431, compared to their free drug or uncrosslinked micelle counterparts. These data suggest that 


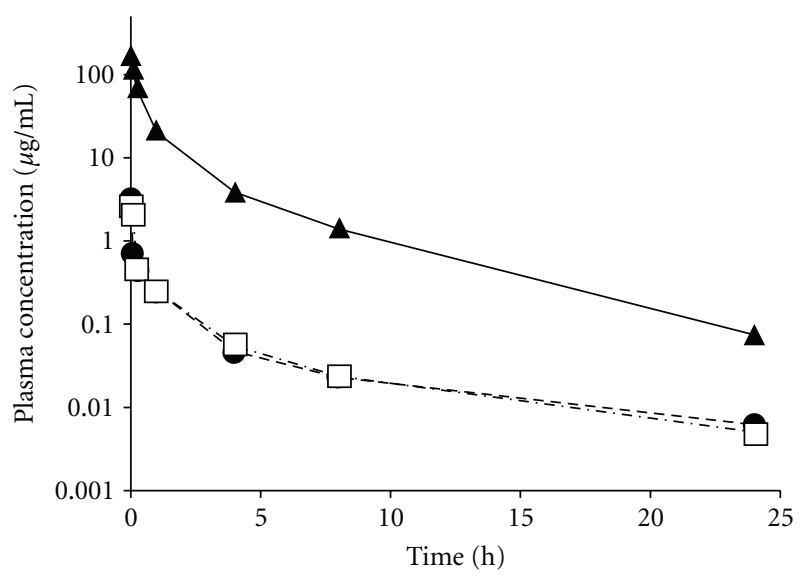

- - Daunorubicin

$-\square-$ Uncrosslinked

$\neg$ Crosslinked

\begin{tabular}{|c|c|c|}
\hline Administration & AUC $(\mu \mathrm{g}-\mathrm{h} / \mathrm{mL})$ & $C_{\max }(\mu \mathrm{g} / \mathrm{mL})$ \\
\hline Crosslinked & 116.39 & 153.82 \\
\hline Uncrosslinked & 1.48 & 2.61 \\
\hline Daunorubicin & 1.3 & 3.29 \\
\hline
\end{tabular}

FIGURE 5: Pharmacokinetics of daunorubicin-loaded micelles in rats. Sprague-Dawley rats were given a single intravenous administration of crosslinked daunorubicin micelle, uncrosslinked daunorubicin micelle, or free daunorubicin at a $10 \mathrm{mg} / \mathrm{kg}$ dose. Plasma was analyzed for daunorubicin concentration at various timepoints. The table depicts the area under curve (AUC) and $C_{\max }$ values for each test article.

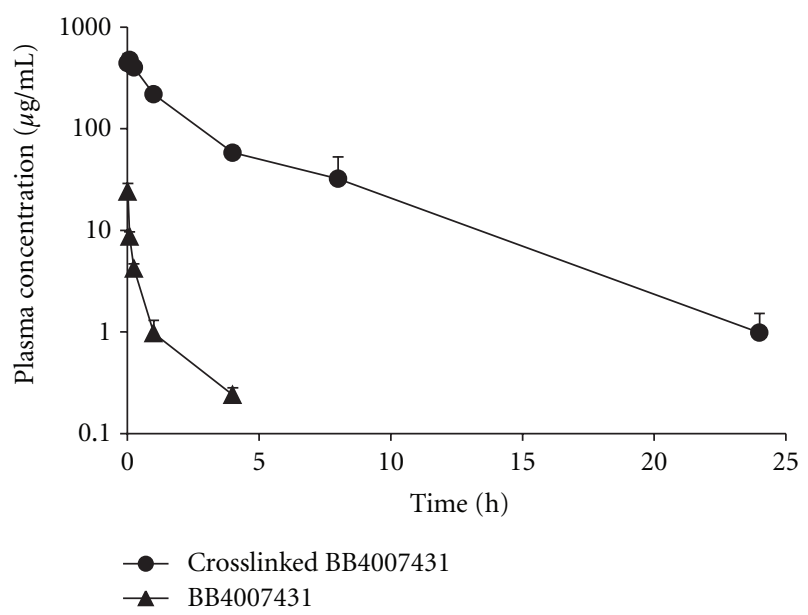

\begin{tabular}{|c|c|c|}
\hline Administration & AUC $(\mu \mathrm{g}-\mathrm{h} / \mathrm{mL})$ & $C_{\max }(\mu \mathrm{g} / \mathrm{mL})$ \\
\hline $\begin{array}{l}\text { Crosslinked } \\
\text { BB4007431 }\end{array}$ & 1196.38 & 476.46 \\
\hline BB4007431 & 5.91 & 24 \\
\hline
\end{tabular}

Figure 6: Pharmacokinetics of crosslinked BB4007431 micelles in rats. Sprague-Dawley rats were given a single intravenous administration of crosslinked BB4007431 micelle, or free BB4007431 at a $25 \mathrm{mg} / \mathrm{kg}$ dose. Plasma was analyzed for BB4007431 concentration at various timepoints. The table depicts the area under curve (AUC) and $C_{\max }$ values for each test article. higher tumor accumulation, and correspondingly improved antitumor efficacy, would be achieved following administration of crosslinked micelle compared to free drug in mouse biodistribution experiments. This would primarily be due to passive targeting by the EPR effect although active targeting has the potential to even further improve delivery of crosslinked micelles.

Polymer micelles hold great promise as drug delivery agents. Indeed, many polymer micelles carrying chemotherapeutic drugs are currently in clinical trials $[6,34]$. The utility of a single platform to encapsulate and systemically deliver hydrophobic cancer drugs allows for faster drug screening and facilitated manufacturing processes. In addition to improving the delivery of current anticancer drugs, the polymer micelle system presented herein holds promise for the development of potent, but insoluble novel anticancer drugs. It is envisioned that this new technology will ultimately provide superior treatment options for patients with cancer.

\section{Conclusions}

A polymer micelle drug delivery system was developed that demonstrated encapsulation and stabilization of a wide variety of hydrophobic anticancer drugs. Drug release from stabilized micelles was determined to be $\mathrm{pH}$ dependent in vitro. In vivo pharmacokinetic studies validated increased stability of crosslinked micelles in biological media and demonstrated improved AUC and $C_{\max }$ compared to uncrosslinked micelles or free drug. These data demonstrate the utility and versatility of a single platform to enable delivery of hydrophobic anticancer drugs to solid tumors.

\section{References}

[1] A. Jemal, R. Siegel, J. Xu, and E. Ward, "Cancer statistics, 2010," CA Cancer Journal for Clinicians, vol. 60, no. 5, pp. 277$300,2010$.

[2] V. P. Torchilin, "Micellar nanocarriers: pharmaceutical perspectives," Pharmaceutical Research, vol. 24, no. 1, pp. 1-16, 2007.

[3] H. Maeda, T. Sawa, and T. Konno, "Mechanism of tumortargeted delivery of macromolecular drugs, including the EPR effect in solid tumor and clinical overview of the prototype polymeric drug SMANCS," Journal of Controlled Release, vol. 74, no. 1-3, pp. 47-61, 2001.

[4] H. Maeda, J. Wu, T. Sawa, Y. Matsumura, and K. Hori, “Tumor vascular permeability and the EPR effect in macromolecular therapeutics: a review," Journal of Controlled Release, vol. 65, no. 1-2, pp. 271-284, 2000.

[5] K. Kataoka, T. Matsumoto, M. Yokoyama et al., "Doxorubicinloaded poly(ethylene glycol)-poly( $\beta$-benzyl-L-aspartate) copolymer micelles: their pharmaceutical characteristics and biological significance," Journal of Controlled Release, vol. 64, no. 1-3, pp. 143-153, 2000.

[6] D. Sutton, N. Nasongkla, E. Blanco, and J. Gao, "Functionalized micellar systems for cancer targeted drug delivery," Pharmaceutical Research, vol. 24, no. 6, pp. 1029-1046, 2007.

[7] E. R. Gillies and J. M. J. Fréchet, "pH-responsive copolymer assemblies for controlled release of doxorubicin," Bioconjugate Chemistry, vol. 16, no. 2, pp. 361-368, 2005. 
[8] N. Nasongkla, X. Shuai, H. Ai et al., "cRGD-functionalized polymer micelles for targeted doxorubicin delivery," Angewandte Chemie International Edition, vol. 43, no. 46, pp. 63236327, 2004.

[9] J. Luo, K. Xiao, Y. Li et al., "Well-defined, size-tunable, multifunctional micelles for efficient paclitaxel delivery for cancer treatment," Bioconjugate Chemistry, vol. 21, no. 7, pp. 1216$1224,2010$.

[10] C. Zheng, L. Qiu, X. Yao, and K. Zhu, "Novel micelles from graft polyphosphazenes as potential anti-cancer drug delivery systems: drug encapsulation and in vitro evaluation," International Journal of Pharmaceutics, vol. 373, no. 1-2, pp. 133-140, 2009.

[11] D. Kim, E. S. Lee, K. T. Oh, Z. Gao, and Y. H. Bae, "Doxorubicin-loaded polymeric micelle overcomes multidrug resistance of cancer by double-targeting folate receptor and early endosomal pH," Small, vol. 4, no. 11, pp. 2043-2050, 2008.

[12] R. Savić, T. Azzam, A. Eisenberg, and D. Maysinger, "Assessment of the integrity of poly(caprolactone)-b-poly(ethylene oxide) micelles under biological conditions: a fluorogenicbased approach," Langmuir, vol. 22, no. 8, pp. 3570-3578, 2006.

[13] F. Koizumi, M. Kitagawa, T. Negishi et al., "Novel SN-38incorporating polymeric micelles, NK012, eradicate vascular endothelial growth factor-secreting bulky tumors," Cancer Research, vol. 66, no. 20, pp. 10048-10056, 2006.

[14] D. Vetvicka, M. Hruby, O. Hovorka et al., "Biological evaluation of polymeric micelles with covalently bound doxorubicin," Bioconjugate Chemistry, vol. 20, no. 11, pp. 2090-2097, 2009.

[15] G. S. Kwon, M. Yokoyama, T. Okano, Y. Sakurai, and K. Kataoka, "Biodistribution of micelle-forming polymer-drug conjugates," Pharmaceutical Research, vol. 10, no. 7, pp. 970974, 1993.

[16] J. Du and S. P. Armes, "pH-responsive vesicles based on a hydrolytically self-cross-linkable copolymer," Journal of the American Chemical Society, vol. 127, no. 37, pp. 12800-12801, 2005.

[17] J. O. Kim, G. Sahay, A. V. Kabanov, and T. K. Bronich, "Polymeric micelles with ionic cores containing biodegradable cross-links for delivery of chemotherapeutic agents," Biomacromolecules, vol. 11, no. 4, pp. 919-926, 2010.

[18] C. J. Rijcken, C. J. Snel, R. M. Schiffelers, C. F. van Nostrum, and W. E. Hennink, "Hydrolysable core-crosslinked thermosensitive polymeric micelles: synthesis, characterisation and in vivo studies," Biomaterials, vol. 28, no. 36, pp. 5581-5593, 2007.

[19] K. Qi, Q. Ma, E. E. Remsen, C. G. Clark Jr., and K. L. Wooley, "Determination of the bioavailability of biotin conjugated onto shell cross-linked (SCK) nanoparticles," Journal of the American Chemical Society, vol. 126, no. 21, pp. 6599-6607, 2004.

[20] Y. Chan, T. Wong, F. Byrne, M. Kavallaris, and V. Bulmus, "Acid-labile core cross-linked micelles for $\mathrm{pH}$-triggered release of antitumor drugs," Biomacromolecules, vol. 9, no. 7, pp. 1826-1836, 2008.

[21] X. Hu, X. Chen, J. Wei, S. Liu, and X. Jing, "Core crosslinking of biodegradable block copolymer micelles based on poly (ester carbonate)," Macromolecular Bioscience, vol. 9, no. 5, pp. 456-463, 2009.

[22] X. Shuai, T. Merdan, A. K. Schaper, F. Xi, and T. Kissel, "Core-cross-linked polymeric micelles as paclitaxel carriers," Bioconjugate Chemistry, vol. 15, no. 3, pp. 441-448, 2004.
[23] K. Breitenkamp, K. Sill, and H. Skaff, "Poly(ethylene glycol) containing chemically disparate end groups," US Patent no. 7, $560,588,2009$.

[24] J. R. Kramer and T. J. Deming, "General method for purification of $\alpha$-amino acid-N-carboxyanhydrides using flash chromatography," Biomacromolecules, vol. 11, no. 12, pp. 36683672, 2010.

[25] H. R. Kricheldorf, "Polypeptides and 100 years of chemistry of $\alpha$-amino acid N-carboxyanhydrides," Angewandte Chemie International Edition, vol. 45, no. 35, pp. 5752-5784, 2006.

[26] G. A. Eby, "Zinc ion availability-the determinant of efficacy in zinc lozenge treatment of common colds," Journal of Antimicrobial Chemotherapy, vol. 40, no. 4, pp. 483-493, 1997.

[27] J. Liu, H. Lee, and C. Allen, "Formulation of drugs in block copolymer micelles: drug loading and release," Current Pharmaceutical Design, vol. 12, no. 36, pp. 4685-4701, 2006.

[28] H. Gelderblom, J. Verweij, K. Nooter, and A. Sparreboom, "Cremophor EL: the drawbacks and advantages of vehicle selection for drug formulation," European Journal of Cancer, vol. 37, no. 13, pp. 1590-1598, 2001.

[29] Y. Bae, W. D. Jang, N. Nishiyama, S. Fukushima, and K. Kataoka, "Multifunctional polymeric micelles with folate-mediated cancer cell targeting and $\mathrm{pH}$-triggered drug releasing properties for active intracellular drug delivery," Molecular BioSystems, vol. 1, no. 3, pp. 242-250, 2005.

[30] N. Nasongkla, E. Bey, J. Ren et al., "Multifunctional polymeric micelles as cancer-targeted, MRI-ultrasensitive drug delivery systems," Nano Letters, vol. 6, no. 11, pp. 2427-2430, 2006.

[31] V. P. Torchilin, "Targeted pharmaceutical nanocarriers for cancer therapy and imaging," AAPS Journal, vol. 9, no. 2, article 15, pp. E128-E147, 2007.

[32] X. L. Wu, J. H. Kim, H. Koo et al., "Tumor-targeting peptide conjugated $\mathrm{pH}$-responsive micelles as a potential drug carrier for cancer therapy," Bioconjugate Chemistry, vol. 21, no. 2, pp. 208-213, 2010.

[33] K. M. Laginha, E. H. Moase, N. Yu, A. Huang, and T. M. Allen, "Bioavailability and therapeutic efficacy of HER2 scFvtargeted liposomal doxorubicin in a murine model of HER2overexpressing breast cancer," Journal of Drug Targeting, vol. 16, no. 7-8, pp. 605-610, 2008.

[34] Y. Matsumura and K. Kataoka, "Preclinical and clinical studies of anticancer agent-incorporating polymer micelles," Cancer Science, vol. 100, no. 4, pp. 572-579, 2009. 

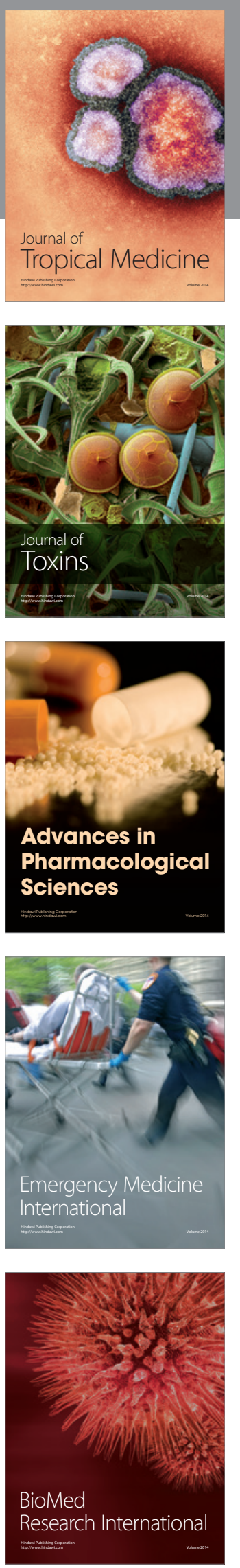
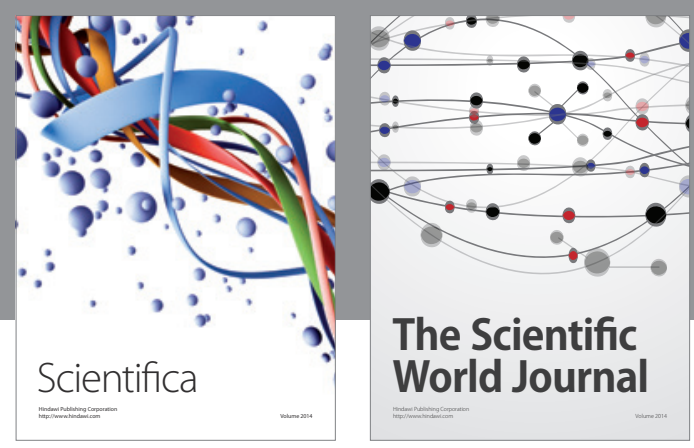

The Scientific World Journal
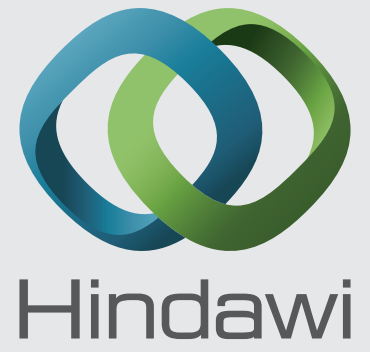

Submit your manuscripts at

http://www.hindawi.com
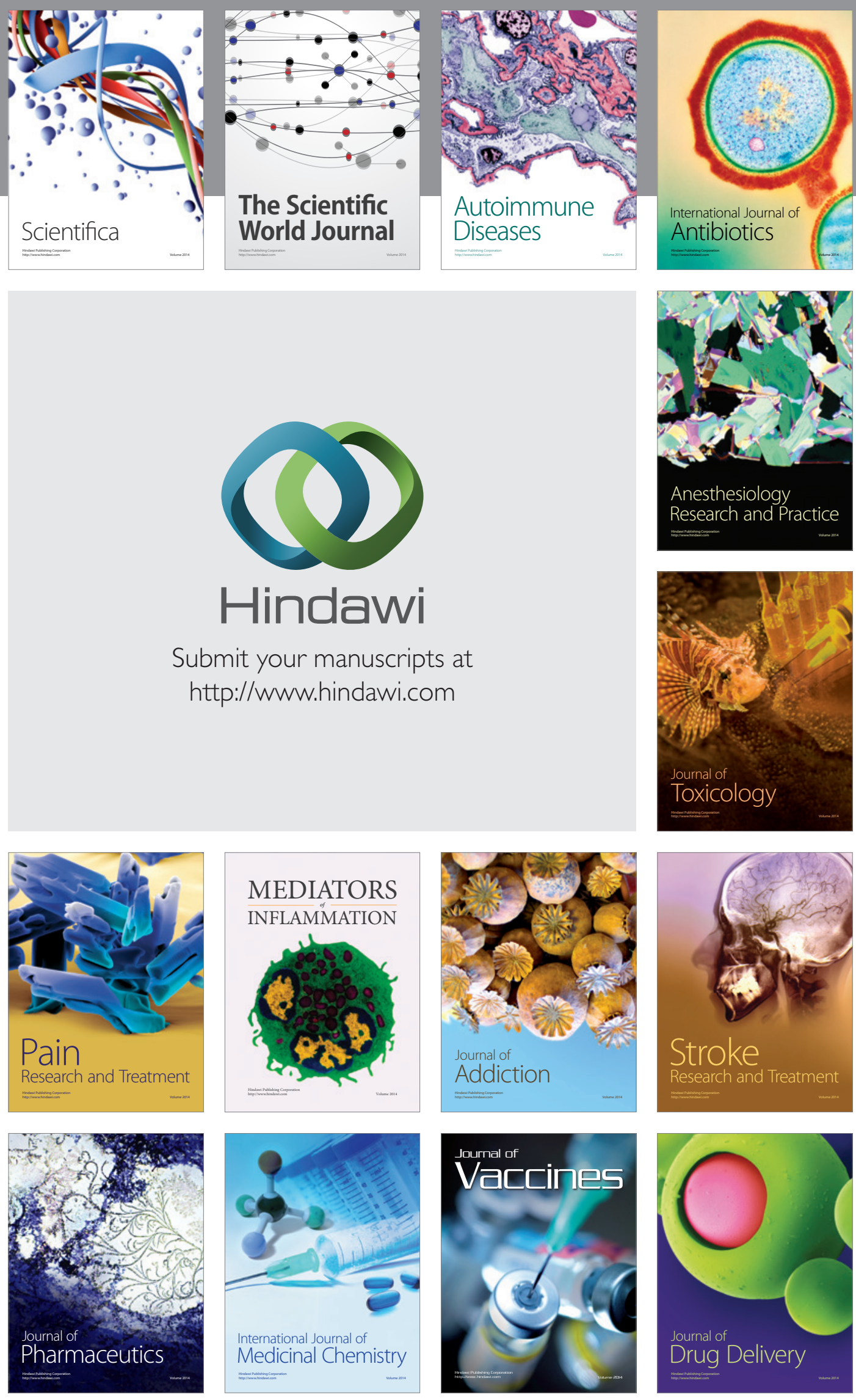